\title{
Estimating Coverage of Hepatitis B Birth Dose Vaccination: A Pilot Study in Western Pacific Countries
}

\author{
Anthony Burton* \\ Department of Immunization, Vaccines and Biologicals, World Health Organization, 20 avenue Appia, 1211 Geneva 27, Switzerland
}

\begin{abstract}
Background: The universal administration of a birth dose of the hepatitis B vaccine within the first 24 hours of life is crucial for preventing perinatal hepatitis B virus transmission and can significantly reduce the disease burden of chronic hepatitis B infection. Unlike with the majority of vaccines recommended by WHO, there is currently no methodology to generate hepatitis $B$ birth dose coverage estimates.
\end{abstract}

Methods: Methods used by the WHO and UNICEF for estimating coverage for other vaccines were expanded to include indicators that allow validation of timeliness of administered hepatitis B birth doses in Western Pacific Countries. These indicators include percentages of births with skilled attendance or in health facilities and differences between WHO/UNICEF estimates and country-reported coverage for other vaccines.

Results: We made hepatitis B birth dose estimates for 23 countries between 1999 and 2010. Estimates for the 2010 birth cohort ranged from 99\% (eight countries) to as low as $2 \%$ (Viet Nam. Estimates for ten of 23 countries different from data reported by national authorities for at least one year. In some countries, the variability was as great as $50 \%$. In several instances, estimates incorporating indicator data were different from those generated by the standard WHO/UNICEF protocol.

Conclusions: A protocol for estimating hepatitis B birth dose coverage has been proposed and we have shown that supplemental indicator data can provide useful validation. Extrapolation to other regions will require availability of similar data.

Keywords: Hepatitis B; World Health Organization (WHO); Hepatocellular carcinoma; Immunization

\section{Introduction}

Perinatal hepatitis B virus (HBV) transmission is a major determinant of HBV carrier status and is strongly associated with HBV prevalence, particularly hepatitis $\mathrm{B}$ e antigen $(\mathrm{HBeAg})$ prevalence in childbearing women, and with vaccine coverage levels of hepatitis $B$ birth dose [1]. For the prevention of perinatal and early horizontal transmission of HBV, the World Health Organization (WHO) recommends the universal administration of a birth dose of hepatitis $B$ vaccine (HepB) within the first 24 hours of life [2]. While approximately $90 \%$ of infants infected perinatally become chronic carriers, the risk for chronic HBV infection decreases to $30 \%$ for children infected between ages one and four years and to less than $5 \%$ for persons infected as adults [3-5]. Chronic HBV infection manifests predominantly as liver cirrhosis or hepatocellular carcinoma, for which treatment is limited in many countries. Recent prevalence estimates suggest that globally 240 million peopleare chronically infected with HBV [6].

The recombinant HepB consisting of purified hepatitis B surface antigen (HBsAg) was first introduced in 1986 and has since replaced the earlier plasma-derived hepatitis $\mathrm{B}$ vaccine originally released in 1982. The recombinant HepB is now incorporated in many different combinations vaccines, such as a pentavalent diphtheria, tetanus, pertussis, haemophilus influenza type B, and HepB (DTPHibHepB) combination vaccine and is also available in a monovalent formulation. HepB has been administered over a billion times and has both a very strong safety [7] and efficacy record [8-13]. Several country studies are available showing the effectiveness of $\mathrm{HepB}$ vaccination in reducing prevalence of chronic HBV infection, for example from Alaska [14], the Gambia [15], Italy [16], and Taiwan [17]. Prevention of early infection, achieved by a birth dose of $\mathrm{HepB}$, is the principle intervention to reduce the disease burden attributable to chronic HBV infection.
To ensure maximum efficacy, the HepB birth dose must be administered as soon as possible following birth, preferably within 24 hours [2]. Currently 88 WHO member states recommend universal administration of a HepB birth dose. However, definitions of a birth dose vary by country and current recording and reporting practices do not necessarily permit direct estimation of the proportion of births receiving a dose of HepB within the first 24 hours of life. Since the birth dose may be administered through multiple strategies by a variety of personnel, the development of vaccination policies and the establishment of accurate and complete recording and reporting systems may require significant coordination.

Since $2000 \mathrm{WHO}$ and UNICEF jointly provide annual estimates of national infant immunization coverage for selected vaccines. Levels and trends of immunization coverage are used (i) to monitor the performance of immunization services locally, nationally and internationally; (ii) to guide strategies for the eradication, elimination and control of vaccinepreventable diseases; and (iii) to identify areas of immunization systems that may require additional resources and focused attention. Models of vaccine-preventable disease burden frequently include immunization coverage levels among their components [18]. While coverage estimates are available for the principle recommended infant vaccines,

*Corresponding author: Anthony Burton, Department of Immunization, Vaccines and Biologicals, World Health Organization, 20 avenue Appia, 1211 Geneva 27 , Switzerland, E-mail: BurtonA@who.int

Received March 18, 2013; Accepted March 24, 2013; Published March 26, 2013

Citation: Burton A (2013) Estimating Coverage of Hepatitis B Birth Dose Vaccination: A Pilot Study in Western Pacific Countries. J Vaccines Vaccin 4: 178 doi:10.4172/2157-7560.1000178

Copyright: (c) 2013 Burton A. This is an open-access article distributed under the terms of the Creative Commons Attribution License, which permits unrestricted use, distribution, and reproduction in any medium, provided the original author and source are credited. 
including the third dose of hepatitis B vaccine (HepB3), estimates are not currently made for the birth dose of hepatitis $B$ vaccine.

Our objective is to produce estimates of HepB birth dose coverage for all WHO member states of the Western Pacific Region. We extend the methods used to produce coverage estimates for other infant vaccines by including additional data from surveys and databases. Validation procedures are applied using appropriate proxy indicators, e.g. percentage of births in health facilities. We believe this method can be used to generate HepB birth dose coverage for all other countries.

\section{Materials and Methods}

To estimate the proportion of births receiving a dose of $\mathrm{HepB}$ within 24 hours of life we searched for and extracted relevant data and applied methods previously used to estimate vaccine coverage. Data used include: national immunization coverage for the final dose in the HepB series (HepB3) and BCG reported by national health authorities to WHO and UNICEF (WHO Vaccine Preventable Disease Monitoring System, WHO/IVB) [19], immunization coverage survey results from the Demographic and Health Surveys (DHS) [20], UNICEF Multiple Indicator Surveys [21], and National EPI surveys. We also conducted an additional non-systematic MEDLINE search to obtain studies and reports that address НеpB birth dose coverage. In order to validate obtained information, we extracted estimates for the percentage of births occurring in health facilities and percentage of births with skilled attendance from the WHO Regional Office for the Western Pacific Region's (WPR) Tool (now published as the Health Information and Intelligence Platform for the Western Pacific Region, Interim Solution) [22], the WPR Country Health Information Profiles (CHIP) for 20092011 [23], Millennium Development Goals Indicators [24], EPI Profiles: 2009, and Operational indicators for hepatitis B control [25] (Figure 1).

We assumed that survey data are more accurate than countryreported reported data unless there are clear flaws in the survey methodology or report e.g. sample size not stated [18]. If survey data were available on delayed birth doses, i.e. $>24$ hours after birth, we assume that the proportion of birth doses that was administered late was the same for children whose coverage was based on caretaker recall. If only the timely birth dose is reported, we assume that all caretaker recall birth dose are timely.

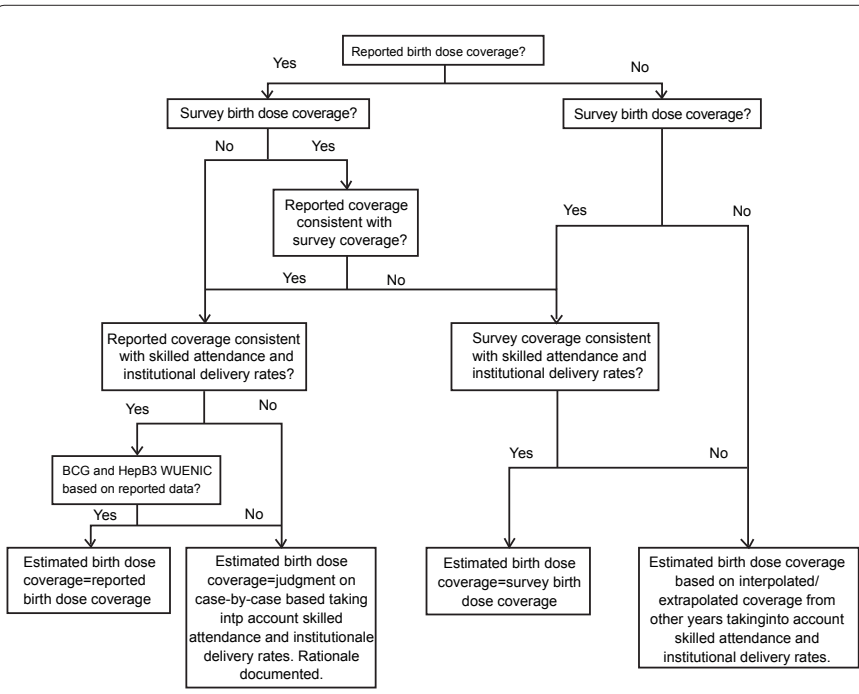

Figure 1: Methodological approach to estimate HepB birth dose coverage.

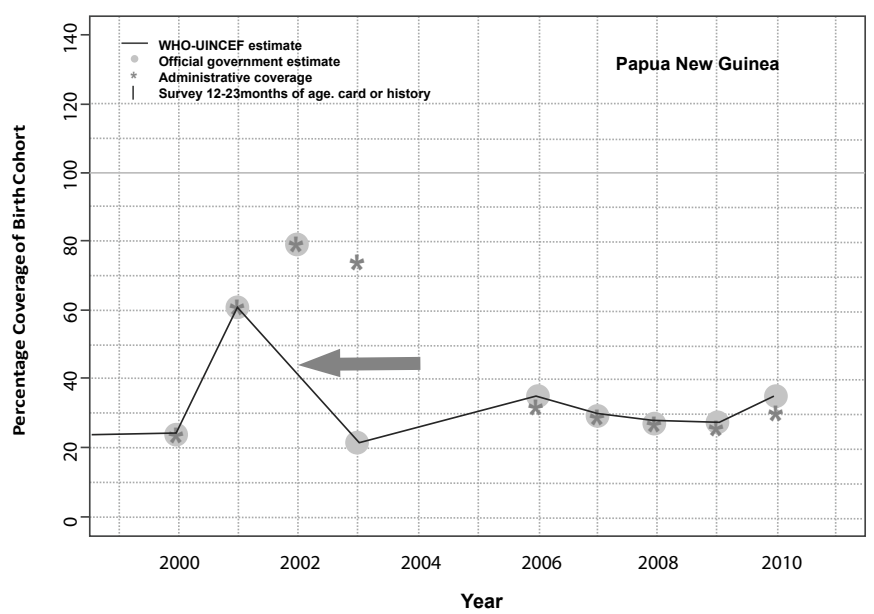

Figure 2: Hepatitis B birth dose coverage for Papua New Guinea: preliminary estimates based on reported coverage using interpolation for unreported years. The arrow shows where indicator data contradicts official country estimate.

Proxy indicators used for validating reported birth dose coverage include coverage reported by national authorities and WHO and UNICEF estimates of coverage (WUENIC) for HepB3 and BCG. If WUENIC estimates for HepB3 and BCG are not based on coverage reported by national authorities, we assume that inaccurate or inconsistent immunization coverage reporting applies to reported birth dose coverage as well. If nationally reported coverage varies significantly over time, we assume this reflects as reporting artefact rather than true sudden changes in birth dose coverage.

Since birth dose administration requires a skilled health care professional, it is highly unlikely that birth dose coverage exceeds the percentage of births attended by a skilled attendant. These births may occur in health facilities or at home, if a country has a mechanism for administering the birth dose at home, as indicated on the EPI profile. If the percentage of institutional births reported by a country is $100 \%$, we assume no births take place outside of institutions.

\section{Results}

We estimate that in 2010 eight countries reached 99\% coverage but the lowest coverages were as little in 2\% (Viet Nam) and 9\% (Lao). Overall, for 10 of 23 countries in the Western Pacific region, our methodology provided preliminary birth dose coverage estimates that were different to those reported by national authorities. The variability was as great as $50 \%$ (Kiribati, 2003). Noteworthy are countries where the indicator data either supported or contradicted the reported data. Figures 2-5 present four examples for different outcomes and implications using this methodology.

\section{HepB birth dose estimate is based on estimates reported by national authorities}

Between 2006 and 2010, data reported by national authorities indicate consistent coverage in Papa New Guinea and, as there is no birth dose survey data, the preliminary estimate of HepB birth dose coverage is based entirely on reported data. Survey data available for other vaccines generally supported reported data in Papua New Guinea over this time period, suggesting reported birth dose coverage in 20062010 is reliable.

A greater variation between reported data in 2001 and 2002 implies a 
high likelihood of inaccurate reporting, which is furthermore indicated by the fact that the gives an official estimate that is vastly lower than their administrative data. In addition, the reported birth dose coverage (79\%) percentage exceeds that of institutional births (44.8\%). As Papua New Guinea does not have a mechanism for administration of the birth dose at home, this suggests that for at least 2002 the official country report is too high. The 2002 estimate of $41 \%$ interpolated between 2001 and 2003 reported data is supported by the supplementary indicator data (as indicated by the arrow in figure 2).

It is noteworthy that the survey assessing the 2004 birth cohort reported $15.5 \%$ coverage within 24 hours or $26 \%$ within 48 hours. Administration after 24 hours does not qualify as a valid dose, but $26.6 \%$ coverage is comparable to the later reported coverage estimates in 2006-2010 (ranging 27\%-35\%) which indicates that the country may potentially be using an incorrect definition of the birth dose in these earlier years too. This survey was not included in the preliminary estimate because it only included information from immunization cards and not parental recall data for estimating the timely administration coverage.

\section{HepB birth dose estimates influenced by WUENIC estimates for other infant vaccines}

For Kiribati, no proxy indicator data such as skilled attendance at birth or institutional birth indicator data were available, but there were proxy indicator data on the difference between country-reported data and WUENIC for other vaccines, namely BCG coverage.

As figure 3 shows, between 2000 and 2004 the official country estimate and administrative data varied widely. Following the standard WUENIC guidelines, no estimate is made exceeding 99\%. However, the proxy indicator data gives additional information to suggest reporting issues in this country. In addition to variability seen with the reported coverage of other vaccines, the reported HepB birth dose coverage exceeds that of BCG (2002 and 2003, as shown in figure 3 by the arrows). As BCG does not have stringent criteria for timeliness, it seems unlikely that the administration of the HepB birth dose was both within 24 hours and at a greater coverage than BCG.

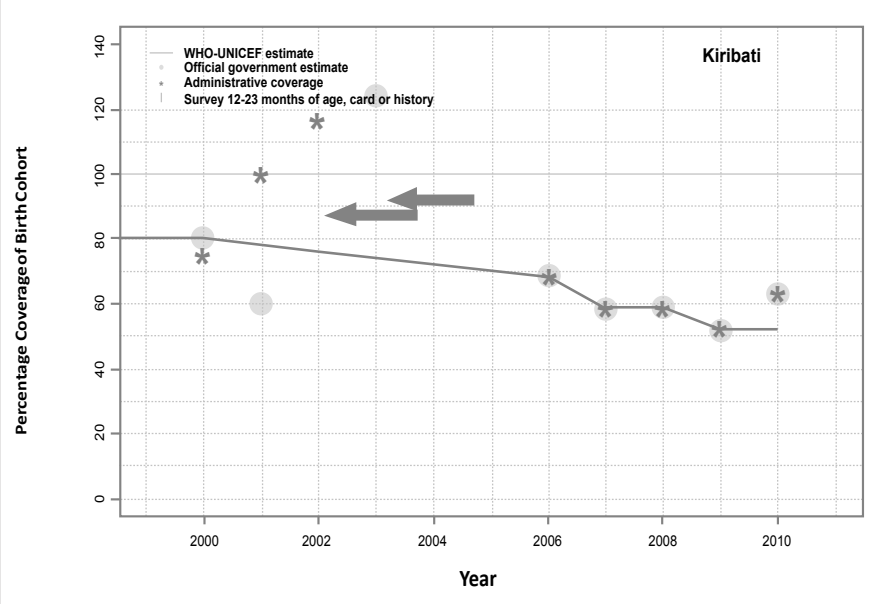

Figure 3: Hepatitis B birth dose coverage for Kiribati: preliminary estimates based on country-reported coverage using interpolation for unreported years. The arrows show years where BCG coverage was below country-reported HepB birth dose coverage (88\% in 2002 and $91 \%$ in 2003).

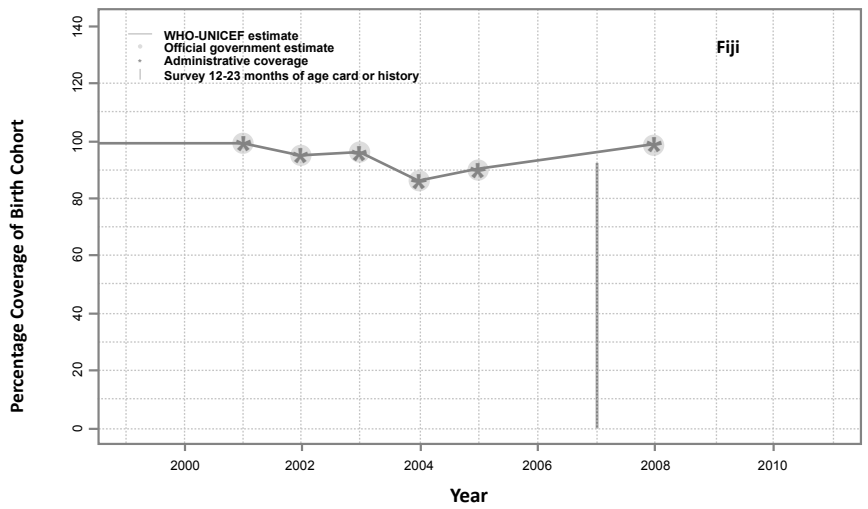

Figure 4. Hepatitis B birth dose coverage for Fiji: preliminary estimates based on reported coverage using interpolation for unreported years. Births in health facilities were higher than reported coverage of birth dose in all years.

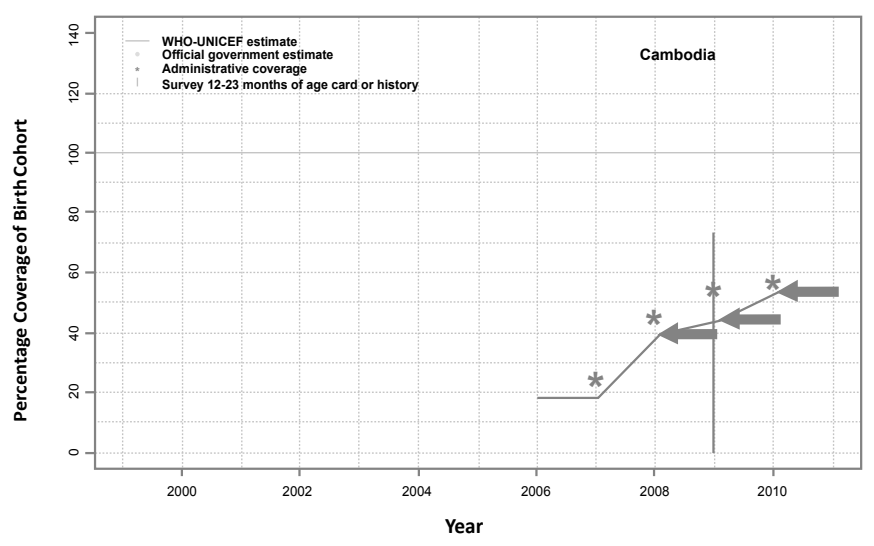

Figure 5. Hepatitis B birth dose coverage for Cambodia: preliminary estimates based on country administrative data but 'capped' by indicator data on the percentage of births in health facilities. The arrows shows where indicator data 'caps' the estimate, as indicated by the WHO-UNICEF line, which would have otherwise been calculated based on country's administrative data (39\% in $2008,44 \%$ in $2009,54 \%$ in 2010, CHIP).

\section{HepB birth dose estimates based on nationally reported data and survey data}

Ideally, country birth dose coverage estimates can be based on both data reported by national authorities and survey results. Fiji provides an example where survey coverage estimates are available for one cohort and although slightly lower than the interpolated reported data, generally corroborate this official estimate. The same survey suggests coverage for all other vaccines were conversely under-reported for this cohort.

\section{HepB birth dose estimates capped by indicator data}

In the case of contradiction between indicator data and official country estimates, appropriate and reliable survey data is normally used in the WUENIC method in lieu of a national estimate. If survey data follow the same general trend, as in the case of Cambodia, coverage estimates can be calibrated to the survey for other years too.

However, in this specific Cambodian example, indicator data- as annotated on figure 5 (the arrows)- for percentage of births in health facilities are below both the country-reported and survey-reported birth dose coverage. Although skilled attendance at birth is reported 
to be higher in the CHIP database $(58 \%$ in $2008,63 \%$ in $2009,71 \%$ in 2010), Cambodia does not have a policy of administering the birth dose at home births so these extra births outside health facilities with skilled attendance are still unlikely to have been reached with the birth dose in a timely manner. This suggests the reported as well as the survey data from which the WUENIC estimate would normally be based, is likely erroneous for these years. The percentage of births occurring in health facilities 'caps' the coverage estimate in our methodology (39\% in 2008, $44 \%$ in 2009 and $54 \%$ in 2010).

\section{Discussion}

For the first time, a protocol for generating estimates of HepB birth dose coverage has been outlined. We evaluated whether the use of the proxy indicators improves the estimation process or whether the WUENIC methodology currently utilized for other vaccines would be equally viable for generating HepB birth dose estimates. Looking at country examples, Papua New Guinea illustrated how indicator data can corroborate interpolated estimates leading to the decision to reject the country-reported estimate in 2002. Similarly, proxy indicator data support a lower estimate than reported by the country for Kiribati in 2002 and 2003. In countries where country-reported or survey data were accepted, indicator data either validates this choice (e.g. Fiji) or questions a new estimate, such as in Cambodia. The example of Cambodia particularly illustrates the utility of this new methodology as indicator data directly challenges the output of the WUENIC method in 2008-2010 and caps the estimate in a way that would previously not have been proposed. This is strong evidence to support the use of data on facility births and skilled attendance at birth to improve the estimate process on a country-by-country basis.

The current analysis contributes significantly to the goal of estimating global country-specific HepB birth dose coverage comparable to other vaccine coverage estimates in terms of documented methodology and comprehensiveness. In developing an estimation process and approach for the Western Pacific countries, we believe that there is potential for use of this methodology to produce estimates for other countries that administer the birth dose. However, there are scarce data on health facilities and skilled attendance birth in some regions, e.g. Africa, and the proposed estimation process may not be appropriate in the absence of indicator data. By means of the proposed method, countries that are in need of support, e.g. in terms of strengthening systems of administering, reporting birth dose, and in terms of logistics of birth dose delivery can be identified.

\section{Addressing main challenges and limitations}

HepB birth dose coverage estimates are not available from most surveys such as DHS, MICS or EPI. Surveys on immunization coverage often record the percentage coverage for the birth dose as validated by a card or by parental recall, which can help assessing whether the dose was valid for inclusion in the WHO and UNICEF estimation process. Too often, however, surveys do not include sufficiently detailed questions on HepB immunization to differentiate between a valid birth dose and simply the first dose, which may be given later at first clinical contact. Previous experience has shown that even when HepB immunization is included in the questionnaire, data collected are often not presented in the final report

So far, many countries have not established robust mechanism for confirming timeliness of birth dose and uncertainty is associated with using parental recall on timeliness of administration. The example of Fiji indicates that parental recall is not sensitive enough to distinguish between timely and late doses. However, it could also be argued that parental recall is more accurate with a birth dose than with vaccines administered later in life because it is the only vaccine to be given in first 24 hours and may be more memorable. On the other hand, BCG scarifies and provides validation of immunization card or parental reported coverage. Nonetheless, the unusual timing of the HepB birth dose could help explain why a survey may seem more consistent for birth dose than for other EPI vaccines (e.g. in Fiji).

Although there are potential explanations for discrepancies between data reported by national authorities and surveys or indicators, a direct country consultation can provide further insight into the selection of one competing data source of another. From our experience with immunization coverage reporting, there are numerous reasons- such as hospital records not being reliable or general issues with denominators when calculating administrative coverage, which would not be an issue with a survey. This was illustrated in the example of Kiribati (Figure 3) where some reported coverage were over $100 \%$, suggesting denominator issues when calculating coverage i.e. inaccurate information on the size of the birth cohort. Problems with denominators also cause extreme fluctuation in reported coverage in small countries such as the Pacific Islands and in countries with high proportion of home births, as it is not apparent whether administrative data include non-facility births. An effective birth notification system with the potential to link it to other data sources would be a major step in accomplishing reliable vaccine coverage estimates but is also crucial for other health data. This would overcome limitations of surveys that are related to cohort effects in a way that cohort-specific estimates can be produced.

Another important issue refers to national administrative definitions of timeline of birth dose. Annual changes can affect available data used for generating coverage estimates. This fact may have triggered the sudden changes in reported coverage in example 1 for Papa New Guinea. Estimates from Vietnam also allude to changes in policy on birth dose administration. Initially administration within three days of birth was considered as a birth dose and is thus inaccurate following the latter definition of a birth dose to be given within 24 hours of birth. For validation purpose, the percentage of these birth doses that were given within 24 hours is a crucial input into the coverage estimation process and can provide a more realistic estimate than the countryreported data that referred to doses administered within three days. These examples point to the need to record time of birth, which is also relevant for other public health research areas.

Based on our analysis, indicator data can provide relevant validation in generating $\mathrm{HepB}$ birth dose estimates. Extrapolation to other regions will require availability of such data, including surveys which incorporate birth dose questions. The HepB birth dose is important as it is the only vaccine for preventing perinatal transmission, which is associated with a greatly increased risk of developing a chronic HBV infection. Evaluation of coverage is therefore vital for impact assessment and monitoring the efficacy of HepB immunization as a component of a comprehensive strategy to address the burden of disease associated with HBV infection.

\section{Acknowledgements}

We would like to thank Marta Gacic-Dobo, Diana Chang-Blanc, Steven Wiersma, Karen Hennessey, Jorge Mendoza Aldana and Rudi Eggers for providing technical guidance on potentially relevant surveys and country-specific advice.

\section{References}

1. Ott JJ, Stevens GA, Wiersma ST (2012) The risk of perinatal hepatitis B virus transmission: hepatitis $\mathrm{B}$ e antigen $(\mathrm{HBeAg})$ prevalence estimates for all world regions. BMC Infectious Diseases 12: 131 
Citation: Burton A (2013) Estimating Coverage of Hepatitis B Birth Dose Vaccination: A Pilot Study in Western Pacific Countries. J Vaccines Vaccin 4: 178. doi:10.4172/2157-7560.1000178

2. (2009) Hepatitis B vaccines. Wkly Epidemiol Rec 84: 405-419.

3. McMahon BJ, Alward WL, Hall DB, Heyward WL, Bender TR, et al. (1985) Acute hepatitis B virus infection: relation of age to the clinical expression of disease and subsequent development of the carrier state. J Infect Dis 151: 599-603.

4. Beasley, Hwang (1991) Overview of the epidemiology of hepatocellular carcinoma. In: Hollinger FB, Lemon SM, Margolis HS, eds. Viral hepatitis and liver disease Proceedings of the 1990 international symposium on viral hepatitis and liver disease: contemporary issues and future prospects. Baltimore: Williams and Wilkins 532-535.

5. Hyams KC (1995) Risks of chronicity following acute hepatitis B virus infection a review. Clin Infect Dis 20: 992-1000.

6. Ott JJ, Stevens GA, Groeger J, Wiersma ST (2012) Global epidemiology of hepatitis B virus infection: new estimates of age-specific HBsAg seroprevalence and endemicity. Vaccine 30: 2212-2219.

7. GACVS Global Vaccine Safety: Hepatitis B.

8. Xu ZY, Liu CB, Francis DP, Purcell RH, Gun ZL, et al. (1985) Prevention of perinatal acquisition of hepatitis $B$ virus carriage using vaccine: preliminary report of a randomized, double-blind placebo-controlled and comparative trial. Pediatrics 76: 713-718.

9. Zhu QR, Gu XH, Duan SC, Xu HF (1994) Long-term immunogenicity and efficacy of recombinant yeast derived hepatitis $B$ vaccine for interruption of mother-infant transmission of hepatitis B virus. Chin Medl J (Engl) 107: 915918

10. Fortuin M, Chotard J, Jack AD, Maine NP, Mendy M, et al. (1993) Efficacy of hepatitis $B$ vaccine in the Gambian expanded programme on immunisation. Lancet 341: 1129-1131.

11. André FE, Zuckerman AJ (1994) Review: protective efficacy of hepatitis B vaccines in neonates. J MedVirol 44: 144-151.

12. del Canho R, Grosheide PM, Mazel JA, Heijtink RA, Hop WC, et al. (1997) Ten-year neonatal hepatitis B vaccination program, The Netherlands, 19821992: protective efficacy and long-term immunogenicity. Vaccine 15: 16241630.
13. Grading Table III with key references. Conclusion: (i) high-quality evidence to support effectiveness of a primary series of hepatitis B vaccine to prevent any HBV infection at 15 years post-vaccination of infants; (ii) high-quality evidence to support effectiveness of a primary series of hepatitis $B$ vaccine to prevent chronic HBV infection at 15 years post-vaccination of infants; (iii) low-quality evidence to support the effectiveness of a primary series of hepatitis $B$ vaccine to prevent HBV infection at up to 22 years post-vaccination of infants.

14. Harpaz R, McMahon BJ, Margolis HS, Shapiro CN, Havron D, et al. (2000) Elimination of new chronic hepatitis $B$ virus infections: results of the Alaska immunization program. J Infect Dis 181: 413-418.

15. Viviani S, Jack A, Hall AJ, Maine N, Mendy M, et al. (1999) Hepatitis B vaccination in infancy in The Gambia: protection against carriage at 9 years of age. Vaccine 17: 2946-2950.

16. Da Villa G, Piccinino F, Scolastico C, Fusco M, Piccinino R, et al. (1998) A Long term epidemiological survey of hepatitis $B$ virus infection in a hyperendemic area (Afragola, southern Italy): results of a pilot vaccination project. Res Virol 149: 263-270.

17. Hsu HM, Lu CF, Lee SC, Lin SR, Chen DS (1999) Seroepidemiologic survey fo hepatitis $B$ virus infection in Taiwan: the effect of hepatitis $B$ mass immunization. J Infect Dis 179: 367-370.

18. Burton A, Monasch R, Lautenbach B, Gacic-Dobo M, Neill M, et al. (2009) WHO and UNICEF estimates of national infant immunization coverage: methods and processes. Bull World Health Organ 87: 535-541.

19. WHO Preventable Diseases Monitoring System, WHO/IVB

20. MEASURE DHS Demographic and Health Surveys.

21. UNICEF. Multiple Indicator Cluster Survey, Statistics and Monitoring

22. World Health Organization Western Pacific Region: Data by health topic.

23. World Health Organization Western Pacific Region: Countries and Areas Accessed from:

24. United Nations Statistics Division: Millennium Development Goals Indicators

25. National immunization data- EPI summaries by country. World Health Organization Western Pacific Region: Expanded Programme on Immunization. 